\title{
Prototipo de vivienda con comodidad térmica por paneles solares y generador eólico para zonas andinas
}

\author{
Ing. Percy José Vignes Aubert (a) \\ Fis. Rolando Lozano Cusi ${ }^{(b)}$ \\ (a) Empresa Vignes Electronics SAC -_pvignes1@hotmail.com telf. 999855916 \\ (b)Universidad Andina del Cusco - rlozano@uandina.edu.pe - telf. 974976640.
}

\section{RESUMEN}

El objetivo de este trabajo de investigación es presentar un prototipo de vivienda con comodidad térmica por energía solar y generador eólico para las zonas andinas del Perú; mediante innovaciones tecnológicas que brinden un nuevo modelo de vivienda mejorada de manera termodinámica, para contrarrestar los efectos del friaje en dichas zonas, aprovechando la energía solar y eólica que brinda autonomía energética. Además de ofrecer un modelo de vivienda antisísmica y que mejore la calidad de vida de los pobladores.

PALABRAS CLAVE: casa solar con comodidad térmica.

\begin{abstract}
The objective of this research is to present a prototype of housing with thermal comfort with solar energy and wind generator for the andean zones of Peru; with technological innovations that provide a new model of thermodynamically improved housing, to counteract the effects the cold season in these areas, taking advantage of solar and wind energy that provides energy autonomy. In addition to offering an anti-seismic housing model and improving the quality of life of the settlers.
\end{abstract}

KEYWORD: House Solar thermal comfort. 


\section{INTRODUCCIÓN}

En las zonas andinas se presentan las épocas del friaje, que originan bajas temperaturas con víctimas mortales y un gran número de pobladores con enfermedades bronco-pulmonares. Se ha determinado, que gran parte del problema son las viviendas inadecuadas para soportar el intenso frío de la zona. Como consecuencia de este clima, los pobladores de la zona no tienen buenos hábitos de higiene, lo que contribuye a desarrollar enfermedades dermatológicas y gastrointestinales.

En la actualidad el modelo de vivienda en la zona andina, principalmente rurales, presenta las características siguientes:

- No tienen estructuras antisísmicas,

- No cuentan con servicios higiénicos adecuados.

- No tienen comodidad térmica, ni luminosa dentro de la vivienda.

- No tienen agua potable en la vivienda.

- Presentan bichos e insectos en los techos e interiores a la vivienda.

\section{PLANTEAMIENTO}

El proyecto plantea la construcción de un prototipo de vivienda de $50 \mathrm{~m}^{2}$ de área interior, con material noble, en la Comunidad Campesina de Occoruro - Provincia de Anta, Región Cusco - Perú, que ofrezca lo siguiente:

1.- Estructura antisísmica con la utilización de material noble,

2.- Comodidad térmica en el interior de la vivienda durante todo el año. (Más de $18{ }^{\circ} \mathrm{C}$ ).

3.- Comodidad luminosa en el interior de la vivienda. (Más de 200 lux).

4.- Agua en servicios básicos instalados dentro de la vivienda.

5.- Energía eléctrica $220 \mathrm{~V} \mathrm{CA}$, para luminarias y tomacorrientes, generada por paneles solares.

6.- Sistema de comodidad térmica de $18{ }^{\circ} \mathrm{C}$ (calefacción), programable y automatizada en el interior de la vivienda, proporcionado por la energía eléctrica de paneles solares y generador eólico (48 V CD).

7.- Bajo costo de construcción de la vivienda. 


\section{FUENTE DE FINANCIAMIENTO}

1.- Innovateperu (Ministerio de la Producción, Convenio 016-PNICP-FIDECOMPIMEN-2015): S/. 148502.00

2.- Vignes Electronics SAC: S/. 63530.00

3.- Universidad Andina del Cusco: S/. 7000.00

\section{MATERIALES Y MÉTODOS DE CONSTRUCCIÓN}

Las etapas del proceso de construcción son:

1.- Cimentación.- Etapa de preparado del terreno y la cimentación de fierro y concreto (losa), de acuerdo al expediente técnico con diseño antisísmico. (Ver foto 1).

2.- Paredes.- Levantamiento de muros con ladrillo.

Una vez terminado el proceso de cimentación y sobrecimentación, se procede al levantado de las paredes con ladrillo. (Ver foto 2).

En el levantamiento de paredes, se considera la instalación de las ventanas y puertas, que tienen un diseño mejorado en su termodinámica de acuerdo a la distribución establecida en los planos.

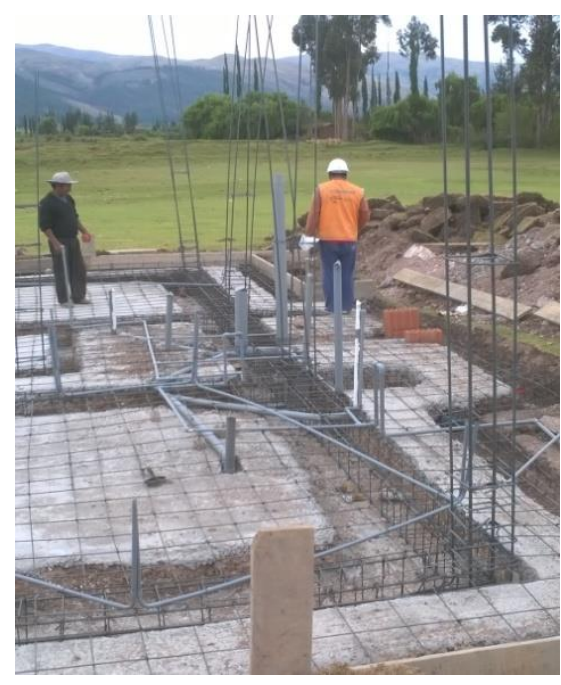

Foto 1.- Estructura antisísmica de vivienda (Foto: Vignes Electronics SAC)

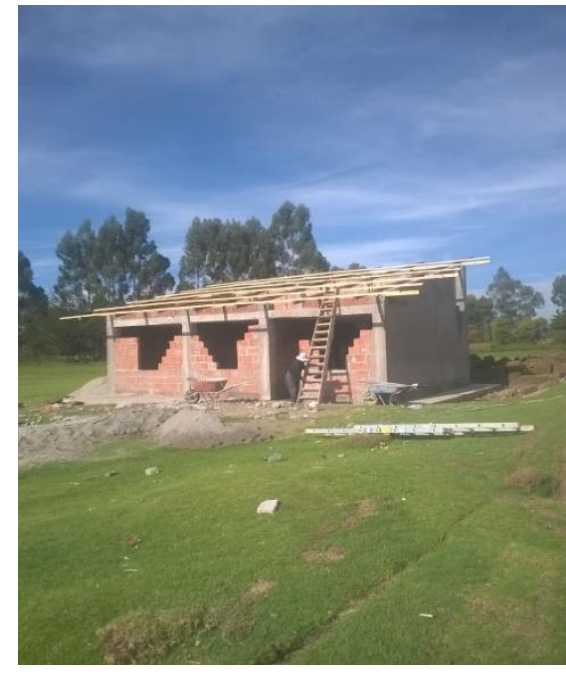

Foto 2.- Construcción con material noble (Foto: Vignes Electronics SAC) 
3.- Techo.- En la etapa del techado, se realizó el vaciado con concreto, se prepararon las vigas de madera y demás estructuras para el techado (una agua), tanto para soportar las calaminas como para el soporte de los paneles solares instalados encima de la lámina corrugada, teniendo en cuenta el cableado respectivo. (Ver foto 3).

Para las viguetas y demás soportes del techo se utilizó madera pino que no se deforma y permite un armado preciso, además presenta un mejor acabado.

El techo inclinado tiene una pendiente de 15 grados respecto a la horizontal, lo cual permite la mejor perpendicularidad para la absorción de la radiación solar. Esta inclinación se calculó para la ubicación geográfica de la construcción (Anta - Cusco). Ver gráfico 1.

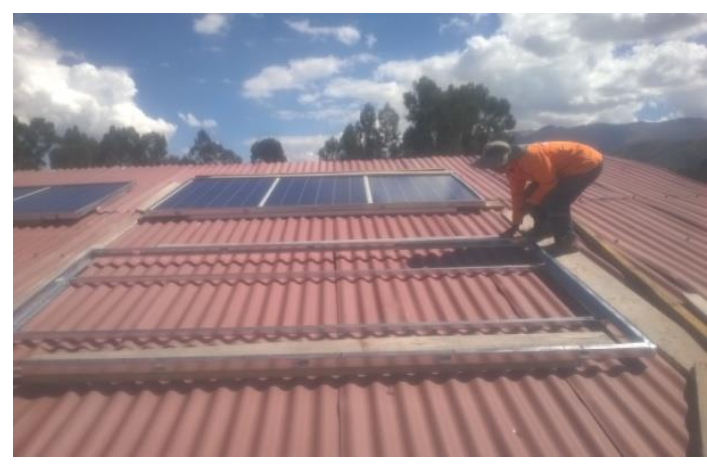

Foto 3.- Instalación de paneles solares fotovoltaicos en el techo (Foto: Vignes Electronics SAC)

4.- Acabado y pintado de muros, esta actividad se realizó luego del estucado de las paredes interiores y exteriores con yeso, enseguida el lijado y masillado de la superficie, finalmente se procedió con el pintado interior y exterior de las paredes.

5.- Puertas y ventanas.- Se confeccionaron las puertas y ventanas de acuerdo con las especificaciones técnicas y planos de la vivienda. Se instalaron un total de 4 puertas y 6 ventanas. 
La puerta exterior tiene en su parte interna material aglomerado (tecnopor) y lana de vidrio como aislamiento térmico y acústico. Las puertas tienen instaladas sus respectivas chapas.

Cada ventana tiene 2 vidrios triples en paralelo, para que cumpla la función de aislamiento térmico y acústico.

6.- Pisos.- La base del piso es de concreto, para lo cual se realiza el vaciado de la losa y vereda, de acuerdo a las especificaciones de mezcla establecidas en el expediente técnico.

Luego del proceso de fraguado del piso, se prepara la etapa de techado.

Cabe destacar que el piso final de la construcción será de madera laminada de $6 \mathrm{~mm}$, para lo cual se requiere que la losa de concreto esté nivelada y pulida con cemento. Al final de todas las etapas se instalan los paneles térmicos confeccionados especialmente para el piso y luego se coloca el acabado en material laminado. (Ver fotos 4,5 y 6 ).

7.- Instalaciones sanitarias.- Las instalaciones sanitarias comprenden los servicios básicos en baño y cocina.

La ducha tiene una poza de fibra de vidrio de fácil instalación, durabilidad y buen acabado.

Las paredes de la ducha tienen revestimiento cerámico color celeste para protección de la humedad y permitir una buena y fácil limpieza.

Del mismo modo, se cuenta con el servicio básico de inodoro y lavatorio de porcelana al interior de la vivienda.

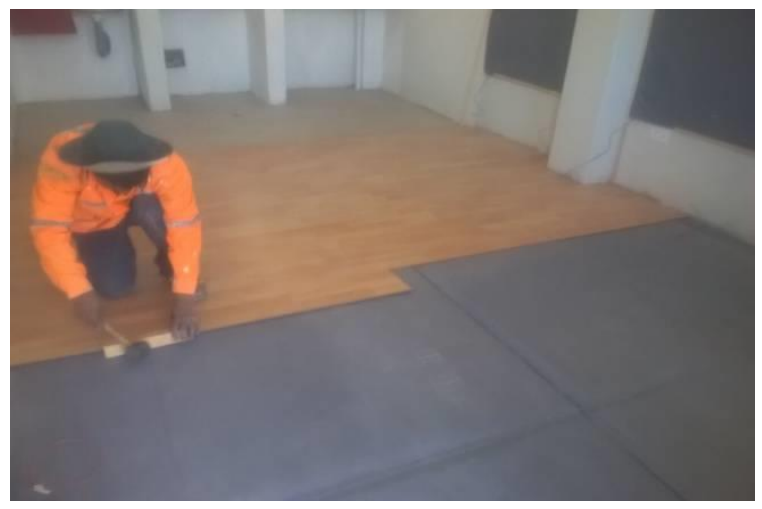

Foto 4.- Instalación de piso laminado encima de paneles térmicos 
(Foto: Vignes Electronics SAC)

8.- Sala de baterías.- Es un ambiente especialmente construido para la instalación del banco de baterías y equipo conversor de energía. Comprende de una sala techada y con puerta de acceso independiente a la vivienda, ventilada para la extracción de los gases generados por las baterías.

9.- Paneles solares.- Se instalaron 12 paneles solares de $250 \mathrm{~W}$ cada uno, encima de las calaminas del techo, las cuales se interconectaron para obtener 3 grupos de suministro de energía de $48 \mathrm{~V} \mathrm{CC}$ a las baterías del mismo modo agrupadas.

10.- Panel de control.- Esta zona está instalada en el pasadizo de la vivienda, donde se encuentran los controladores de carga de las baterías, los pirómetros de control de temperatura y panel eléctrico de control. (Ver foto 8).

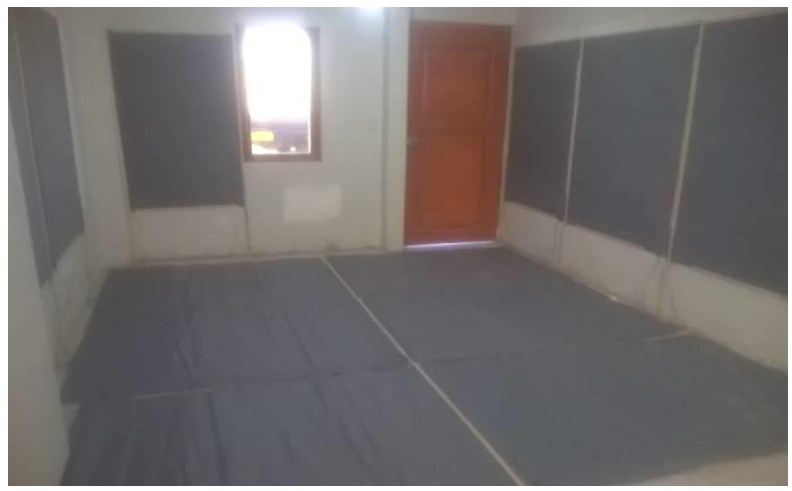

Foto 5.- Instalación de paneles térmicos en paredes y piso. (Foto: Vignes Electronics SAC)

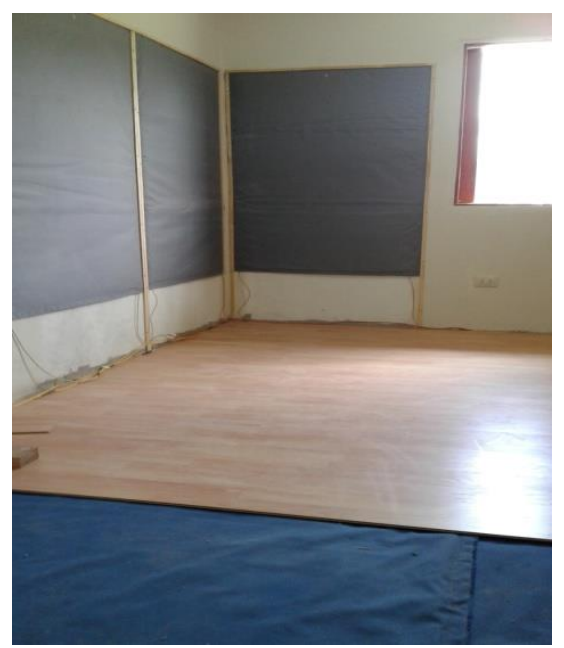

Foto 6.- Instalación de cobertura laminada en piso. (Foto: Vignes Electronics SAC). 


\section{MÉTODO}

La vivienda se ha dividido en 3 zonas cálidas, cada una de ellas tiene un juego de paneles térmicos instalados en las paredes y en piso.

Estos paneles están diseñados para calentar con 48 V CC (Voltios de Corriente Continua).

Los paneles térmicos se han conectado en paralelo en las paredes y en el piso de cada zona de calentamiento y son controlados por pirómetros digitales programables para el control de la temperatura de cada zona. Se ha preferido el uso de la energía CC en los paneles térmicos para disminuir las pérdidas de energía por conversión de $\mathrm{CC}$ a CA.

Los paneles solares proporcionan energía, la que es almacenada en el banco de baterías (12 baterías de 150 Ah cada una) agrupadas en 48 V CC cada una y es alimentado durante el día (ver foto 7). Durante la noche los vientos existentes en la zona hacen funcionar el generador eólico $(3 \mathrm{~kW})$, que proporciona energía que se almacena en las baterías durante la noche. De esta manera, logramos tener suficiente energía en las baterías durante el día y la noche.

Además del sistema de calefacción en la vivienda, se cuenta con un conversor de energía de CC a CA, logrando tener 220 V CA con $1000 \mathrm{~W}$ de energía para las luminarias y artefactos electrodomésticos dentro de la vivienda.

El diseño de los elementos constructivos de la vivienda, contribuyen con la termodinámica en su conjunto, logrando optimizar el gasto de la energía dentro de la vivienda.

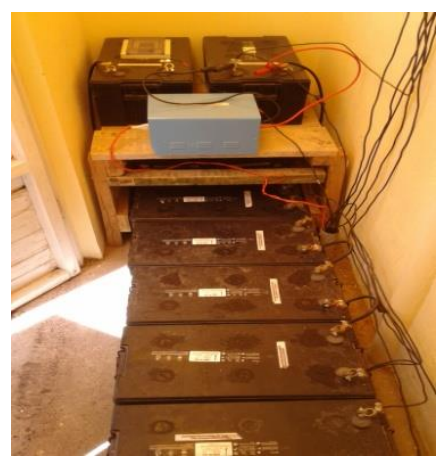

Foto 7.- Banco de baterías

(Foto: Vignes Electronics SAC)

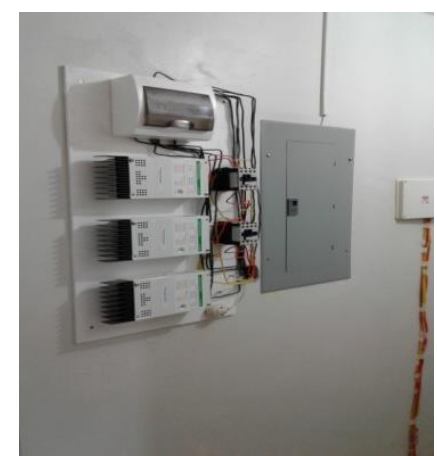

Foto 8.- Panel control

(Foto: Vignes Electronics SAC) 
Para la validación de resultados de comodidad térmica, luminosidad y humedad, se han tomado mediciones de temperatura en 5 viviendas de la zona y se ha comparado con las mediciones obtenidas en el prototipo de vivienda. (Ver resultados en Anexo 1).

\section{RESULTADOS}

La construcción de la vivienda cuenta con comodidad térmica y luminosa, además de servicios básicos de agua, alcanzando el objetivo del proyecto.

Los niveles de luminosidad al interior de la vivienda son superiores a 200 lx en promedio.

La temperatura promedio al interior de la vivienda es de $20{ }^{\circ} \mathrm{C}$, obtenida con el sistema de calefacción por energía solar y eólica.

\section{DISCUSIÓN}

En proyectos similares de vivienda solar para zonas andinas presentada por la Universidad Nacional de Ingeniería (UNI) y la Pontificia Universidad Católica del Perú (PUCP), se concentra la innovación sobre la entrada a la vivienda — que es uno de los puntos principales por donde ingresa el frío-. En dicho proyecto se incorpora un invernadero en el acceso a la vivienda; que es un ambiente tipo antesala hecho de material plástico y que permite calentarse por el sol e irradiar su energía calorífica al interior a través de la puerta principal cuando está abierta.

Este modelo tiene como desventaja la poca iluminación interior a la vivienda, puesto que su diseño no contempla estas mejoras.

El proyecto que presentamos tiene por objetivo mejorar la comodidad térmica dentro de la vivienda, a través de un sistema eléctrico de calefacción instalado en las paredes y en el piso de los diferentes ambientes mediante energía combinada: solar y eólica. (Ver foto 9).

El sistema regula la atmósfera interior en forma automática, a través de sensores de temperatura instalados en los diferentes ambientes. Se utiliza un juego de pirómetros digitales programables para el control de temperatura.

Parte del sistema de energía es proporcionar energía eléctrica de 220 V CA, para el uso de electrodomésticos básicos dentro de la vivienda y mejorar la calidad de vida del poblador. 


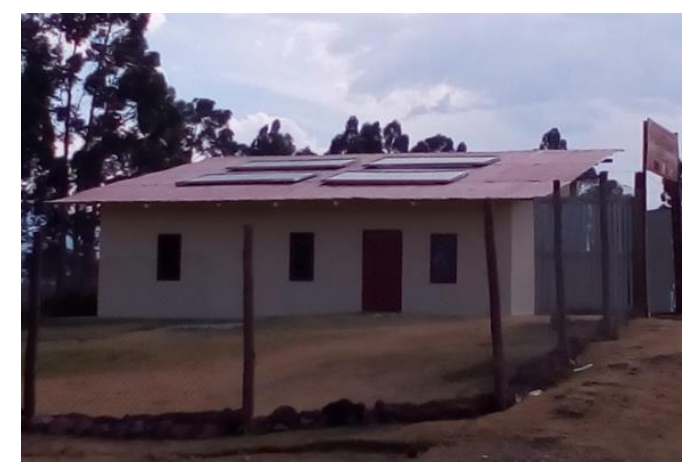

Foto 9.- Juego de paneles solares instalados en el techo con inclinación de $15^{\circ} \mathrm{C}$

(Foto: Vignes Electronics SAC)

\section{CONCLUSIONES}

1.- El modelo de vivienda construido, alcanza los objetivos planteados, como la comodidad térmica y luminosa.

2.- En la ejecución del proyecto, se utilizaron equipos digitales de medición de temperatura y humedad del tipo data logger (adquisición automática de datos), los cuales se utilizaron durante los 12 meses del año, con el fin de medir el comportamiento termodinámico y de humedad de la casa, tanto en su interior como en el exterior.

3.- La luminosidad obtenida al interior de la vivienda eliminó la anidación de bichos, arañas y demás insectos en los techos.

4.- Se requiere monitorear la temperatura y humedad en diferentes épocas del año, con el fin de conseguir resultados definitivos de los objetivos del proyecto.

5.- Este sistema de captación de energía que recoge la energía del sol de manera gratuita, y cuya fuente es inacabable, constituye un gran aporte a la comunidad, mucho más para aquellas poblaciones ubicadas en zonas alto andinas, quienes tendrán la posibilidad de disfrutar de una vivienda confortable, acogedora, con agua corriente para su aseo personal y libre de la presencia de alimañas. Sin duda, constituye una solución a las temporadas del friaje que enfrentan cada cierto tiempo y que en los últimos años se han venido presentando con más frecuencia y en épocas inesperadas. 
6.- Con este prototipo de edificación se cumplen los aspectos principales que todo usuario debe considerar en el planteamiento de selección de un sistema de calefacción convencional, que son los siguientes:

Aplicaciones de energía solar térmica en viviendas unifamiliares

a) Bienestar térmico a través de la obtención de energías renovables.

b) Seguridad: diseño antisísmico.

c) Satisfacción de la demanda energética para uso doméstico.

d) Minimización del consumo energético.

e) Fácil mantenimiento.

f) Protección del medio ambiente.

g) El factor costo.

7.- Además de haber cumplido con los objetivos planteados, es necesario también indicar que este proyecto aporta de manera intrínseca en rebajar la huella ecológica del planeta, que es un requisito planetario que todos estamos en el deber de afrontar. (Ver Foto 10).

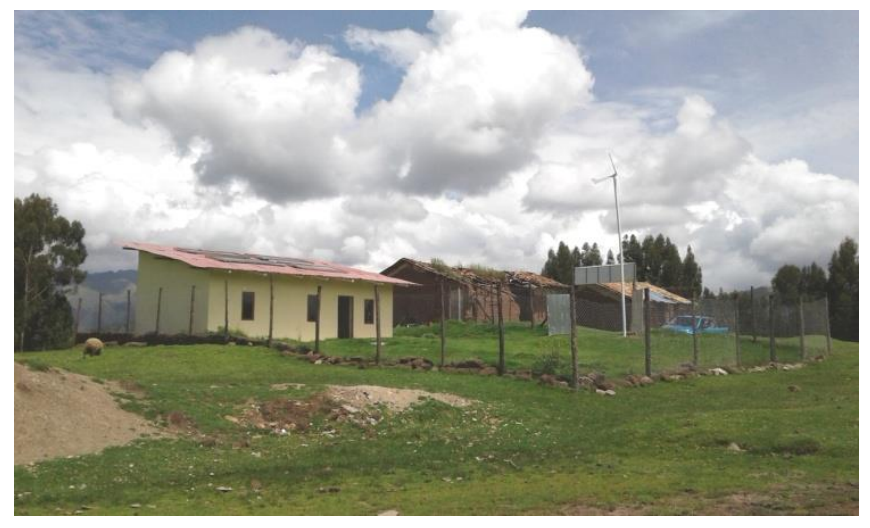

Foto 10.- Casa Solar Eólica terminada. (Foto: Vignes Electronics SAC) 


\section{BIBLIOGRAFÍA}

(CENSOLAR), C. d. (1993). La energía solar: Aplicaciones prácticas. Sevilla, España: Centro de Estudios de la Energia Solar.

ENDEV-PERÚ. (2016). Proyecto Energía, Desarrollo y Vida. Lima, Perú. Obtenido de http://www.endevperu.org.pe/

Gil, G. (Agosto de 2010). Innovaciones tecnológicas utilizando energías renovables. E-Renova Energías renovables para la gente, 3(11). Obtenido de https://www.inti.gob.ar/erenova/restringido/documentos/

Harman Calle, Ú. (2 de Noviembre de 2011). Casa ecológica andina para el desarrollo rural sostenible. (F. E. PUCP, Ed.) América Renovable - Revista Especializada en Energías Renovables y Medio Ambiente, 5(14), 38-42.

Ministerio de Vivienda, C. y. (agosto de 2010).

http://www3.vivienda.gob.pe/dnc/archivos/NOTAS/bioclimatica.pdf. Obtenido de http://www3.vivienda.gob.pe/dnc/archivos/NOTAS/bioclimatica.pdf: http://www3.vivienda.gob.pe/dnc/archivos/NOTAS/bioclimatica.pdf

Ministerio de Vivienda, C. y. (2010). Reglamento Nacional de Edificaciones. (E. "El Peruano", Ed.) Lima. Obtenido de http://www.urbanistasperu.org/rne/pdf/Reglamento\%20Nacional\%20de\%20Edificaciones .pdf 


\section{ANEXO 1:}

Cuadro de valores comparativos en viviendas de la zona y los de la casa solar construida:

\begin{tabular}{|l|c|c|}
\hline Parámetros físicos & $\begin{array}{l}\text { Viviendas de la zona } \\
\text { promedio de 05 viviendas } \\
\text { de la zona) }\end{array}$ & Casa solar prototipo \\
\hline $\begin{array}{l}\text { Temperatura al interior } \\
\left({ }^{\circ} \mathrm{C}\right)\end{array}$ & 11 & 20 \\
\hline $\begin{array}{l}\text { Iluminación promedio } \\
(\text { Lux })\end{array}$ & 90.4 & 300 \\
\hline Humedad relativa $(\%)$ & 75 & 45 \\
\hline
\end{tabular}

Fuente propia: Elaboración de datos tomados con sensores y procesados durante la construcción por el Físico. Rolando Lozano Cusi. (Investigador Universidad Andina del Cusco).

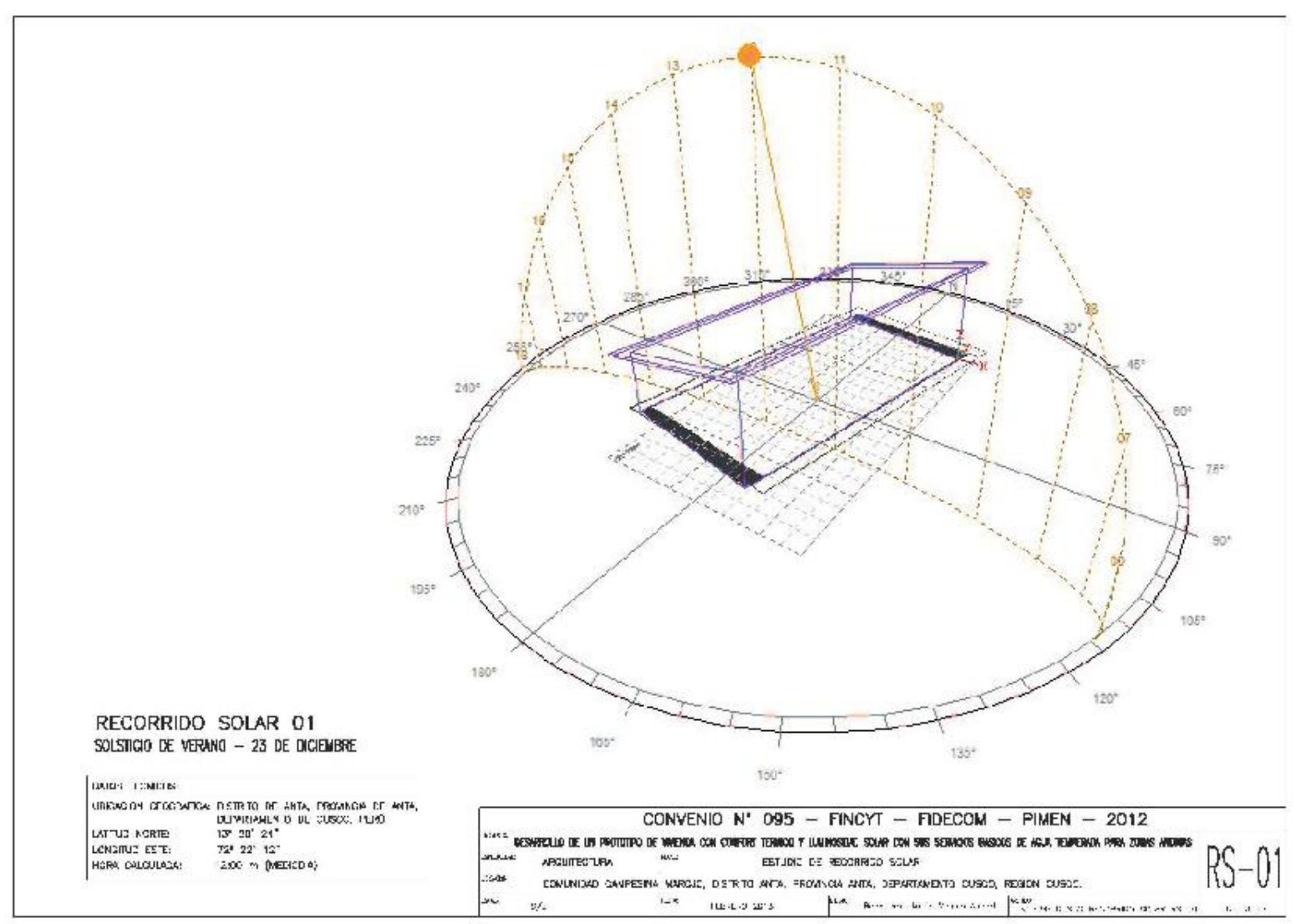

Gráfico 1.- Recorrido solar, inclinación de la casa

(Elaborado: Vignes Electronics SAC) 